\title{
INTEGRAL OPERATOR AND OSCILLATION OF SECOND ORDER ELLIPTIC EQUATIONS
}

\author{
ZHITING XU AND HONGYAN XING
}

Abstract. By using integral operator, some oscillation criteria for second order elliptic differential equation

$$
\sum_{i, j=1}^{d} D_{i}\left[A_{i j}(x) D_{j} y\right]+q(x) f(y)=0, \quad x \in \Omega,
$$

are established. The results obtained here can be regarded as the extension of the well-known Kamenev theorem to Eq. $(E)$.

\section{Introduction and Preliminaries}

We are concerned with the oscillatory behavior of second order elliptic differential equation of the form

$$
\sum_{i, j=1}^{d} D_{i}\left[A_{i j}(x) D_{j} y\right]+q(x) f(y)=0,
$$

where $x=\left(x_{1}, \cdots, x_{d}\right) \in \Omega(a) \subseteq \mathbb{R}^{d}, d \geq 2,|x|=\left[\sum_{i=1}^{d} x_{i}^{2}\right]^{1 / 2}, D_{i} y=\partial y / \partial x_{i}$ for all $i$, $\Omega(a)=\left\{x \in \mathbb{R}^{d}:|x| \geq a\right\}$ for some $a>0$.

Throughout this paper, we assume that the following conditions holds:

$\left(A_{1}\right) \quad A=\left(A_{i j}\right)$ is a real symmetric positive definite matrix function with $A_{i j} \in$ $C_{l o c}^{1+\nu}(\Omega(a), \mathbb{R})$ for all $i, j$, and $\nu \in(0,1)$.

Denote by $u_{\max }(x)$ the largest eigenvalue of the matrix $A$. We suppose that there exists a function $u \in C\left([a, \infty), \mathbb{R}^{+}\right)$such that

$$
u(r) \geq \max _{|x|=r} u_{\max }(x), \quad \text { for } \quad r \geq a ;
$$

$\left(A_{2}\right) q \in C_{l o c}^{\nu}(\Omega(a), \mathbb{R}), \nu \in(0,1)$, and $q(x)$ does not eventually vanish;

$\left(A_{2}\right) f \in C(\mathbb{R}, \mathbb{R}) \cup C^{1}(\mathbb{R}-\{0\}, \mathbb{R}), y f(y)>0$ whenever $y \neq 0$, and $f^{\prime}(y) \geq k>0$ for all $y \neq 0$.

Received September 9, 2002; revised August 26, 2004.

2000 Mathematics Subject Classification. 35B05, 35J60, 34C10.

Key words and phrases. Oscillation, elliptic differential equations, second order, integral operator. 
As usual, a function $y \in C_{l o c}^{2+\nu}(\Omega(a), \mathbb{R}), \nu \in(0,1)$, is called a solution of Eq.(1) if $y(x)$ satisfies Eq.(1) for all $x \in \Omega(a)$. We restrict our attention only the nontrivial solution of Eq.(1), i.e., to the solution $y(x)$ such that $\sup \{|y(x)|: x \in \Omega(b)\}>0$ for every $b \geq a$. Regarding the question of existence of solution of Eq.(1) we refer the reader to the monograph [2]. A nontrivial solution $y(x)$ of Eq.(1) is said to be oscillatory in $\Omega(a)$ if the set $\{x \in \Omega(a): y(x)=0\}$ is unbounded, otherwise it is said to be nonoscillatory. Eq.(1) called oscillatory if all its nontrivial solutions are oscillatory.

Investigation of oscillation of Eq.(1) with variable coefficient $q(x)$ was initiated by Noussair and Swanson [5], who first extended the well-known Fite-Wintner Theorem (see Fite [1] and Wintner [10]) to Eq.(1). As an excellent survey paper, the reader is to recommended to Swanson [6]. Recently, Xu [7], [8] and Zhang et al. [9] have discussed the oscillation property of Eq.(1) under the assumption that the function $q(x)$ is an "integrally small" coefficient, and have shown that most of Kamenev's results in [3] do hold equally well in the case for Eq.(1). However, as far as the authors know there are few results by using integral averaging techniques [4], even through the function $q(x)$ is nonnegative. Motivated by this fact, we intend here to establish Kamenev's integral theorem for oscillation of Eq.(1) based on the integral operator [11]. We are especially interested in case where $q$ may be take on negative values for arbitrarily large $|x|$. Our methodology is somewhat different from that of previous authors, we believe that our approach is simpler and also provides a more unified for the study of Kamenev-type oscillation theorems.

Now, we introduce the general mean and the integral operator.

Following Wong [11], let $D=\{(r, s): r \geq s \geq a\} \in \mathbb{R}^{2}$, and $D_{0}=\{(r, s): r>$ $s \geq a\} \in \mathbb{R}^{2}$. Consider the kernel function $H(r, s) \in C^{1}(D, \mathbb{R})$ such that the following conditions are satisfied:

$\left(H_{1}\right) H(r, r)=0$ for $r \geq a ; H(r, s)>0$ on $(r, s) \in D_{0}$;

$\left(H_{2}\right)$ For each $s \geq a, \lim _{r \rightarrow \infty} H(r, s)=\infty$, and there exists a positive constant $k_{0}$ such that

$$
\begin{gathered}
\lim _{r \rightarrow \infty} \frac{H(r, s)}{H(r, a)}=k_{0}, \text { for all } s \geq a ; \\
\left(H_{3}\right) \frac{\partial H}{\partial s}(r, s) \leq 0,-\frac{\partial H}{\partial s}(r, s)=h(r, s) H(r, s), \text { and } \frac{\partial h}{\partial r}(r, s) \leq 0, \text { for }(r, s) \in D_{0} .
\end{gathered}
$$

Let $\rho \in C^{1}\left([a, \infty), \mathbb{R}^{+}\right)$, we define an integral operator $\Pi_{\tau}^{\rho}$, which is defined in [11], in terms of $H(r, s)$ and $\rho(s)$ as

$$
\Pi_{\tau}^{\rho}(\Theta ; r)=\int_{\tau}^{r} H(r, s) \Theta(s) \rho(s) d s, \quad \text { for } r>\tau \geq a,
$$

where $\Theta \in C([a, \infty), \mathbb{R})$.

For natational simplicity, for arbitrary given functions $\Phi \in C^{1}\left([a, \infty), \mathbb{R}^{+}\right)$and $(u \phi) \in$ $C^{1}([a, \infty), \mathbb{R})$, we define

$$
Q(r)=\Phi(r)\left\{\int_{S_{r}} q(x) d \sigma+\frac{k}{\omega} u(r) \phi^{2}(r) r^{1-d}-[u(r) \phi(r)]^{\prime}\right\}
$$




$$
p(r)=-\left[\frac{\Phi^{\prime}(r)}{\Phi(r)}+\frac{2 k}{\omega} \phi(r) r^{1-d}\right], \quad g(r)=\frac{k r^{1-d}}{\omega u(r) \Phi(r)},
$$

where $S_{r}=\left\{x \in \mathbb{R}^{d}:|x|=r\right\}$ for $r>a, d \sigma$ denotes the spherical integral element in $\mathbb{R}^{d}$, $\omega$ denotes the surface area of unit sphere in $\mathbb{R}^{d}$, i.e., $\omega=2 \pi^{\frac{d}{2}} / \Gamma\left(\frac{d}{2}\right)$.

Lemma 1. [11] Let $H(r, s) \in C^{1}(D, \mathbb{R})$ satisfying $\left(H_{1}\right)-\left(H_{3}\right)$ and $\rho(s) \in C([a, \infty), \mathbb{R})$. Then
(i) $\lim _{r \rightarrow \infty} \frac{1}{H(r, a)} \int_{a}^{r} H(r, s) \rho(s) d s=\infty$, if $\lim _{r \rightarrow \infty} \int_{a}^{r} \rho(s) d s=\infty$;
(ii) $\frac{1}{H(r, a)} \int_{a}^{r} H(r, s) \rho(s) d s$ is nondecreasing in $r$, if $\rho(s) \geq 0$ on $[a, \infty)$.

\section{Main Results}

In this section, we establish Kamenev's integral oscillation criteria for Eq.(1) based on the integral operator $\Pi_{\tau}^{\rho}$. For the sake of simplicity, we always assume that the kernel function $H(r, s)$ satisfies condition $\left(H_{1}\right)-\left(H_{3}\right)$, the integral operator $\Pi_{\tau}^{\rho}$ and functions $Q, p, g$ defined by (2) and (3), respectively.

Theorem 1. Assume that there exist functions $\Phi \in C^{1}\left([a, \infty), \mathbb{R}^{+}\right)$and $(u \phi) \in$ $C^{1}([a, \infty), \mathbb{R})$ such that

$$
\limsup _{r \rightarrow \infty} \frac{1}{H(r, a)} \Pi_{a}^{\tau}\left(Q-\frac{1}{4 g}\left[h+p-\frac{\rho^{\prime}}{\rho}\right]^{2}\right)=\infty .
$$

Then Eq.(1) is oscillatory.

Proof. Let $y(x)$ be a nonoscillatory solution of Eq.(1). Without loss of generality, we may assume that $y(x)>0$ for all $x \in \Omega(a)$. Define

$$
W(x)=\frac{1}{f(y)}\left(A^{\nabla} y\right)(x)
$$

where ${ }^{\nabla} y=\left(D_{1} y, D_{2} y, \cdots, D_{d} y\right)^{T}$ denotes the gradient of $y(x)$. Differentiation (5) and using Eq.(1), we obtain that

$$
\operatorname{div} W(x)=-q(x)-f^{\prime}(y)\left(W^{T} A^{-1} W\right)(x)
$$

Put

$$
Z(r)=\Phi(r)\left[\int_{S_{r}} W(x) \cdot \mu(x) d \sigma+u(r) \phi(r)\right]
$$


where $\mu(x)=x /|x|,(x \neq 0)$, denotes the outward unit normal. By Green's formula in (6), we have

$$
\begin{aligned}
Z^{\prime}(r) & =\frac{\Phi^{\prime}(r)}{\Phi(r)} Z(r)+\Phi(r)\left\{\int_{S_{r}} \operatorname{div} W(x) d \sigma+[u(r) \phi(r)]^{\prime}\right\} \\
& =\frac{\Phi^{\prime}(r)}{\Phi(r)} Z(r)-\Phi(r)\left\{\int_{S_{r}} q(x) d \sigma+\int_{S_{r}} f^{\prime}(y)\left(W^{T} A^{-1} W\right)(x) d \sigma-[u(r) \phi(r)]^{\prime}\right\}
\end{aligned}
$$

In view of $\left(A_{1}\right)$,

$$
\left(W^{T} A^{-1} W\right)(x) \geq u_{\max }^{-1}(x)|W(x)|^{2} .
$$

The Schwartz inequality gives

$$
\int_{S_{r}}|W(x)|^{2} d \sigma \geq \frac{r^{1-d}}{\omega}\left[\int_{S_{r}} W(x) \cdot \mu(x) d \sigma\right]^{2} .
$$

Thus, by (6) and (7), we obtain

$$
\begin{aligned}
Z^{\prime}(r) \leq & \frac{\Phi^{\prime}(r)}{\Phi(r)} Z(r)-\Phi(r)\left\{\int_{S_{r}} q(x) d \sigma+\frac{k r^{1-d}}{\omega u(r)}\left[\int_{S_{r}} W(x) \cdot \mu(x) d \sigma\right]^{2}-[u(r) \phi(r)]^{\prime}\right\} \\
= & \frac{\Phi^{\prime}(r)}{\Phi(r)} Z(r)-\Phi(r)\left\{\int_{S_{r}} q(x) d \sigma+\frac{k r^{1-d}}{\omega u(r)}\left[\frac{Z(r)}{\Phi(r)}-u(r) \phi(r)\right]^{2}-[u(r) \phi(r)]^{\prime}\right\} \\
= & -\Phi(r)\left\{\int_{S_{r}} q(x) d \sigma+\frac{k}{\omega} u(r) \phi^{2}(r) r^{1-d}-[u(r) \phi(r)]^{\prime}\right\} \\
& +\left[\frac{\Phi^{\prime}(r)}{\Phi(r)}+\frac{2 k}{\omega} \phi(r) r^{1-d}\right] Z(r)-\frac{k r^{1-d}}{\omega u(r) \Phi(r)} Z^{2}(r) .
\end{aligned}
$$

Denote $V(r)=Z(r)+\frac{p(r)}{2 g(r)},(8)$ can be rewritten as

$$
Z^{\prime}(r)+g(r) V^{2}(r)+Q(r)-\frac{1}{4} \frac{p^{2}(r)}{g(r)} \leq 0 .
$$

Applying the integral operator $\Pi_{b}^{\rho},(b \geq a)$, to (9), we obtain

$$
\Pi_{b}^{\rho}\left(g V^{2}\right)+\Pi_{\tau}^{\rho}\left(\left[h-\frac{\rho^{\prime}}{\rho}\right] Z\right)+\Pi_{\tau}^{\rho}\left(Q-\frac{p^{2}}{4 g}\right) \leq H(r, b) \rho(b) Z(b) .
$$

Completing squares of $V$ in (10) yields

$$
\Pi_{b}^{\rho}\left(g\left\{V+\frac{1}{2 g}\left[h-\frac{\rho^{\prime}}{\rho}\right]\right\}^{2}\right)+\Pi_{b}^{\rho}\left(Q-\frac{1}{4 g}\left[h+p-\frac{\rho^{\prime}}{\rho}\right]^{2}\right) \leq H(r, b) \rho(b) Z(b) .
$$

Let $b=a$ and divide (11) through by $H(r, a)$. Note the first term is nonnegative, thus

$$
\frac{1}{H(r, a)} \Pi_{a}^{\tau}\left(Q-\frac{1}{4 g}\left[h+p-\frac{\rho^{\prime}}{\rho}\right]^{2}\right) \leq \rho(a) Z(a) .
$$


Take linsup in (12) as $r \rightarrow \infty$. Condition (4) gives a desired contradiction in (12). This proves Theorem 1 .

Remark 1. If we choose the function $\phi(s) \equiv 0$, then Theorem 1 contains Theorem 4 in [5].

Theorem 2. Let $\Phi$ and $\phi$ be as Theorem 1. Assume that there exist functions $\varphi_{i} \in C([a, \infty), \mathbb{R}),(i=1,2)$, such that for $\tau \geq a$,

$$
\lim _{r \rightarrow \infty} \frac{1}{H(r, a)} \Pi_{\tau}^{\rho}\left(\frac{1}{g}\left[h+p-\frac{\rho^{\prime}}{\rho}\right]^{2}\right) \leq \varphi_{1}(\tau)
$$

and

$$
\limsup _{r \rightarrow \infty} \frac{1}{H(r, a)} \Pi_{\tau}^{\rho}(Q) \geq \varphi_{2}(\tau)
$$

where $\varphi_{1}$ and $\varphi_{2}$ satisfy

$$
\lim _{r \rightarrow \infty} \frac{1}{H(r, a)} \Pi_{a}^{\rho}\left(g \rho^{-2}\left[\varphi_{2}-\frac{1}{4} \varphi_{1}\right]_{+}^{2}\right)=\infty
$$

and $[\varphi(r)]_{+}=\max \{\varphi(r), 0\}$. Then Eq.(1) is oscillatory.

Proof. Proceeding as in the proof Theorem 1, we see (11) holds for all $r \geq a$. Divide (11) by $H(r, a)$ and drop the nonnegative first term and obtain

$$
\frac{1}{H(r, a)} \Pi_{\tau}^{\rho}(Q)-\frac{1}{4} \frac{1}{H(r, a)} \Pi_{\tau}^{\rho}\left(\frac{1}{g}\left[h+p-\frac{\rho^{\prime}}{\rho}\right]^{2}\right) \leq \frac{H(r, b)}{H(r, a)} \rho(\tau) Z(\tau) .
$$

Take limsup in above inequality as $r \rightarrow \infty$ and note from (13), (14) and $\left(H_{2}\right)$ that

$$
\varphi_{2}(\tau)-\frac{1}{4} \varphi_{1}(\tau) \leq k_{0} \rho(\tau) Z(\tau)
$$

From which it follows that

$$
k_{0}^{-2} \rho^{-2}(\tau)\left[\varphi_{2}(\tau)-\frac{1}{4} \varphi_{1}(\tau)\right]_{+}^{2} \leq Z^{2}(\tau) .
$$

Then, it follows from (15) and (16) that

$$
\lim _{r \rightarrow \infty} \frac{1}{H(r, a)} \Pi_{a}^{\rho}\left(g Z^{2}\right) \geq \lim _{r \rightarrow \infty} \frac{k_{0}^{-2}}{H(r, a)} \Pi_{a}^{\rho}\left(g \rho^{-2}\left[\varphi_{2}-\frac{1}{4} \varphi_{1}\right]_{+}^{2}\right)=\infty .
$$

Next, we shall show that (17) is not possible.

For (11), set $b=a$. Dividing (11) through $H(r, a)$, and noting (13), (14), we have

$$
\frac{1}{H(r, a)} \Pi_{a}^{\rho}\left(g\left\{V+\frac{1}{2 g}\left[h-\frac{\rho^{\prime}}{\rho}\right]\right\}^{2}\right) \leq \rho(a) Z(a)+\left[\varphi_{2}(b)-\frac{1}{4} \varphi_{1}(b)\right] .
$$


We note that by Lemma 1 (ii), (18) and (13), the following limits exist and finite, that is

$$
\begin{aligned}
& \lim _{r \rightarrow \infty} \frac{1}{H(r, a)} \Pi_{a}^{\rho}\left(g\left\{V+\frac{1}{2 g}\left[h-\frac{\rho^{\prime}}{\rho}\right]\right\}^{2}\right)<\infty, \\
& \lim _{r \rightarrow \infty} \frac{1}{H(r, a)} \Pi_{a}^{\rho}\left(\frac{1}{g}\left[h+p-\frac{\rho^{\prime}}{\rho}\right]^{2}\right)<\infty .
\end{aligned}
$$

Noting that

$$
Z(r)=V(r)-\frac{p(r)}{2 g(r)}=\left(V+\frac{1}{2 g}\left[h-\frac{\rho^{\prime}}{\rho}\right]\right)-\frac{1}{2 g}\left(h+p-\frac{\rho^{\prime}}{\rho}\right) .
$$

Thus

$$
g(s) Z^{2}(r) \leq 2\left\{g(s)\left(V+\frac{1}{2 g}\left[h-\frac{\rho^{\prime}}{\rho}\right]\right)^{2}+\frac{1}{4 g}\left(h+p-\frac{\rho^{\prime}}{\rho}\right)^{2}\right\} .
$$

Consequently, by (19), we obtain

$$
\lim _{r \rightarrow \infty} \frac{1}{H(r, a)} \Pi_{a}^{\rho}\left(g Z^{2}\right)<\infty
$$

which contradicts (17). Therefore, the proof is complete.

Remark 2. Note that Theorem 1 and Theorem 2 do not require conditions $\int^{\infty} \frac{r^{1-d}}{u(r)} d r$ $=\infty$, or $\int_{\Omega(b)} q(x) d x,(b \geq a)$, convergent in [5], [7-9].

In same way as it was done in [11], with an appropriate choice of the functions $H$ and $\rho$, we can derive various oscillation criteria for Eq.(1) from Theorems 1 and 2. For example, define

$$
H(r, s)=(r-s)^{\alpha}, \quad(r, s) \in D, \quad \alpha>1 .
$$

Corollary 1. Let $\alpha>1$. Suppose that the function $g$ is nondecreasing, and

$$
\limsup _{r \rightarrow \infty} r^{-\alpha} \int_{a}^{r}(r-s)^{\alpha}\left\{Q(s)-\frac{p^{2}(s)}{4 g(s)}-\frac{1}{2}\left(\frac{p(s)}{g(s)}\right)^{\prime}\right\} d s=\infty .
$$

Then Eq.(1) is oscillatory.

Proof. Let $\rho(r)=1$ in Theorem 1, observe that

$$
\left[p(s)+\alpha(r-s)^{-1}\right]^{2}=p^{2}(s)+2 \alpha(r-s)^{-1} p(s)+\alpha^{2}(r-s)^{-2} .
$$

By the Bonnet Theorem, for a fixed $r \geq a$ and some $\xi \in[a, r]$, we have

$$
\int_{a}^{r} \frac{(r-s)^{\alpha-2}}{g(s)} d s=\frac{1}{g(a)} \int_{a}^{\xi}(r-s)^{\alpha-2} d s
$$


hence

$$
\lim _{r \rightarrow \infty} \frac{1}{r^{\alpha}} \int_{a}^{r} \frac{(r-s)^{\alpha-2}}{g(s)} d s=0 .
$$

Using integrating by parts, we get

$$
\alpha \int_{a}^{r}(r-s)^{\alpha-1} \frac{p(s)}{g(s)} d s=(r-a)^{\alpha} \frac{p(a)}{g(a)}+\int_{a}^{r}(r-s)^{\alpha}\left(\frac{p(s)}{g(s)}\right)^{\prime} d s .
$$

Thus, Corollary 1 from Theorem 1.

Now, let $\rho(r)=\exp \left(\int_{a}^{r} p(s) d s\right)$ in condition (4), we have

Corollary 2. Let $\alpha>1$. If

$$
\limsup _{r \rightarrow \infty} r^{-\alpha} \int_{a}^{r}(r-s)^{\alpha} Q(s) \exp \left(\int_{a}^{s} p(s) d \tau\right) d s=\infty
$$

and

$$
\lim _{r \rightarrow \infty} r^{-\alpha} \int_{a}^{r}(r-s)^{\alpha-2}[g(s)]^{-1} \exp \left[\int_{a}^{s} p(\tau) d \tau\right] d s<\infty .
$$

Then Eq.(1) is oscillatory.

Remark 3. With the same choice of the functions $H(r, s)$ and $\rho(s)$ as corollary 1 and Corollary 2, more general oscillation criteria for Eq.(1) can be obtained by Theorem 2 similarly. Here we omit the details.

Example 1. Consider the linear elliptic equation of second order

$$
\Delta y+\left(|x|^{\sigma-1} \sin |x|\right) y=0
$$

where $x \in \Omega(1), d=2, \sigma>0, u(r)=1, q(x)=|x|^{\sigma-1} \sin |x|, f^{\prime}(y)=1$ for all $|x|>1$. Choose $\Phi(r)=1, \varphi(r)=0$, then

$$
Q(r)=2 \pi r^{\sigma} \sin r, \quad g(r)=(2 \pi r)^{-1}, \quad p(s)=0 .
$$

For $\sigma>0$, it is easy to verify that

$$
\limsup _{r \rightarrow \infty} \frac{1}{r^{2}} \int_{1}^{r}(r-s)^{2} Q(s) d s=\limsup _{r \rightarrow \infty} \frac{1}{r^{2}} \int_{1}^{r}(r-s)^{2} s^{\sigma} \sin s d s=\infty,
$$

and

$$
\lim _{r \rightarrow \infty} \frac{1}{r^{2}} \int_{1}^{r} \frac{1}{g(s)} d s=\pi .
$$

Hence, Eq.(23) is oscillatory by Corollary 2.

Example 2. Consider the nonlinear elliptic equation of second order

$$
\Delta y+\left(|x|^{v} \cos |x|+\sin |x|\right)\left(y+y^{3}\right)=0,
$$


where $x \in \Omega(1), d=2, v$ is a constant with $2 v>-1, u(r)=1, q(x)=|x|^{v} \cos |x|+\sin |x|$, and $f(y)=y+y^{3}$. Let $\Phi(r)=r^{-1}, \varphi(r)=0$, then

$$
g(r)=(2 \pi)^{-1}, \quad p(r)=r^{-1}, \quad Q(r)=2 \pi\left(r^{v} \cos r+\sin r\right), \quad f^{\prime}(y)=1+y^{2} .
$$

Taking $H(r, s)=(r-s)^{2}$ for $(r, s) \in D$ and $\rho(r)=1$, we have, for all $\tau \geq 1$,

$$
\limsup _{r \rightarrow \infty} \frac{1}{r^{2}} \int_{\tau}^{r}(r-s)^{2} Q(s) d s \geq-2 \pi \tau^{v} \cos \tau-\varepsilon,
$$

and

$$
\lim _{r \rightarrow \infty} \frac{1}{r^{2}} \int_{\tau}^{r} \frac{1}{g(s)}(r-s)^{2}\left[2(r-s)^{-1}+s^{-1}\right]^{2} d s=\frac{2 \pi}{\tau},
$$

where $\varepsilon$ is a positive constant.

Now, set $\varphi_{1}(\tau)=(2 \pi \tau)^{-1}$ and $\varphi_{2}(\tau)=-2 \pi \tau^{v} \cos \tau-\varepsilon$. Then, there exists an integer $N$ such that $(2 N+1) \pi-\frac{\pi}{4}>1$ and if $n \geq N$,

$$
(2 n+1) \pi-\frac{\pi}{4} \leq \tau \leq(2 n+1) \pi+\frac{\pi}{4}, \quad \varphi_{2}(\tau)-\frac{1}{4} \varphi_{1}(\tau) \geq \delta \tau^{v},
$$

where $\delta$ is a small constant. Taking into account that $2 v>-1$, we obtain

$$
\begin{aligned}
\lim _{r \rightarrow \infty} \int_{a}^{r}\left[\varphi_{2}(\tau)-\frac{1}{4} \varphi_{1}(\tau)\right]_{+}^{2} d s & \geq \sum_{n=N}^{\infty} \delta^{2} \int_{(2 n+1) \pi-\frac{\pi}{4}}^{(2 n+1) \pi+\frac{\pi}{4}} s^{2 v} d s \\
& \geq \sum_{n+N}^{\infty} \delta^{2} \int_{(2 n+1) \pi-\frac{\pi}{4}}^{(2 n+1) \pi+\frac{\pi}{4}} s^{-1} d s=\infty
\end{aligned}
$$

This implies that, by Lemma $1(i)$,

$$
\lim _{r \rightarrow \infty} \frac{1}{r^{2}} \int_{a}^{r} \frac{1}{2 \pi}(r-s)^{2}\left[\varphi_{2}(\tau)-\frac{1}{4} \varphi_{1}(\tau)\right]_{+}^{2} d s=\infty .
$$

Accordingly, all conditions of Theorem 2 are satisfied, and hence, Eq.(24) is oscillatory.

\section{References}

[1] W. B. Fite, Concerning the zeros of the solutions of certain differential equations, Trans. Amer. Math. Soc. 19(1918), 341-352.

[2] D. Gilbarg and N. S. Trudinger, Elliptic Partial Differential Equations of Second Order, Spinger-Verlag, Now York, 1983.

[3] I. V. Kamenev, Oscillation of solution of a second order differential equation with an "integrally small" coefficient, Differencial'nye Uravnenija 13(1977), 2141-2148. (In Russian)

[4] I. V. Kamenev, An integral criterion for oscillation of linear differential equations, Math. Zamtki 23 (1978), 249-251. (In Russian)

[5] E. S. Noussair and C. A. Swanson, Oscillation of semilinear elliptic inequalities by Riccati transformation, Canad. J. Math. 32 (1980), 908-923. 
[6] C. A. Swanson, Semilinear second order elliptic oscillation, Cand. Math. Bull, 22(1979), 139-157.

[7] Z. T. Xu, Oscillation of second order elliptic partial differential equations with a "weakly integrally small" coefficient, J. Sys \& Math. Scis 18(1998), 478-484. (In Chinese)

[8] Z. T. Xu, Oscillation of second order nonlinear differential elliptic equations, Appl. Math. JCU (A) 17(2002), 29-36. (In Chinese)

[9] B. G. Zhang, T. Zhao and B. S. Lalli, Oscillation criteria for nonlinear second order elliptic differential equations, Chin Ann of Math(B) 1(1996), 89-102.

[10] A. Wintner, A criterion of oscillatory stability, Quart. J. Appl. Math. 7(1949), 115-117.

[11] J. S. W. Wong, On Kamenev-type oscillation theorems for second-order differential equations with damping, J. Math. Anal. Appl. 258 (2001), 244-257.

Department of Mathematics, South China Normal University, Guangzhou, 510631, P. R. China. E-mail: xztxhyyj@pub.guangzhou.gd.cn

Department of Applied Mathematics, Guangdong University of Technology, Guangzhou, 510090, P. R. China.

E-mail: 928hongy@163.com 